\title{
The Role of Percutaneous Endoscopic Transgastric Jejunostomy in the Management of Enteral Tube Feeding
}

\author{
Ezekiel Wong Toh Yoon
}

\begin{abstract}
Feeding-related adverse events after percutaneous endoscopic gastrostomy (PEG) such as aspiration pneumonia can impede the use of PEG. Percutaneous endoscopic transgastric jejunostomy (PEG-J) using large-bore jejunal tubes with gastric decompression function may improve outcomes by circumventing gastric passage during enteral nutrition and improving drainage of excessive gastric secretions. This report describes a case where PEG-J was successful in maintaining enteral tube feeding in a 72-year-old man when PEG feeding was not tolerated. Patients with unsuccessful PEG feeding can be offered the option of jejunal feeding before terminating enteral nutrition.
\end{abstract}

Keywords: Percutaneous endoscopic gastrostomy; Gastrojejunostomy; Enteral nutrition

\section{Introduction}

Percutaneous endoscopic gastrostomy (PEG) is the method of choice for long-term enteral tube feeding in patients with dysphagia or insufficient oral intake $[1,2]$. Nevertheless, feedingrelated adverse events such as aspiration pneumonia due to gastroesophageal reflux of enteral feed may impede the successful use of PEG. Jejunal (or post-pyloric) feeding may overcome gastric feeding-related adverse events by circumventing gastric passage during enteral nutrition $[3,4]$. Jejunal feeding can be achieved via direct percutaneous endoscopic jejunostomy (D-PEJ) [5] or more commonly by placing a jejunal tube through an existing gastrostomy site, also called a transgastric jejunostomy (PEG-J or JET-PEG) [6]. Jejunal extension tubes placed through PEG tubes are usually more prone to tube dysfunctions such as obstruction or migration into the stomach [7, 8]. Instead of jejunal extension tubes, large-bore jejunal tubes can be inserted directly through the PEG puncture site (not the PEG tube) with the aid of an ultrathin endoscope after removal of the PEG tube [9]. This report describes a case where PEG-J using a jejunal tube with gastric decompression function (pro-

Manuscript accepted for publication May 23, 2016

Department of Internal Medicine, Hiroshima Kyoritsu Hospital, 2-20-20 Nakasu Asaminami-ku, Hiroshima City, Japan. Email: easybs@hotmail.com

doi: http://dx.doi.org/10.14740/gr704w viding simultaneous drainage of gastric secretions) was successful in maintaining enteral tube feeding in a 72-year-old man when PEG feeding was not tolerated.

\section{Case Report}

A 72-year-old man with a previous history of schizophrenia, acute subdural hematoma, duodenal ulcer and paralytic ileus was referred to our hospital for PEG. He has been hospitalized in a psychiatric institution for the past 40 years and has been bed-ridden for the past 5 years due to the subdural hematoma. His oral intake gradually decreased and recurrent episodes of aspiration pneumonia prompted his attending physician to recommend PEG.

Physical examination and blood laboratory markers were unremarkable and he was tube-fed with a nasogastric tube (NGT) for 7 days before undergoing the PEG procedure. Enteral feeding via NGT was well tolerated. Endoscopic imaging before the procedure revealed a deformed duodenal bulb with moderate stenosis (Fig. 1A) and gastric ulcer scars (Fig. 1B).

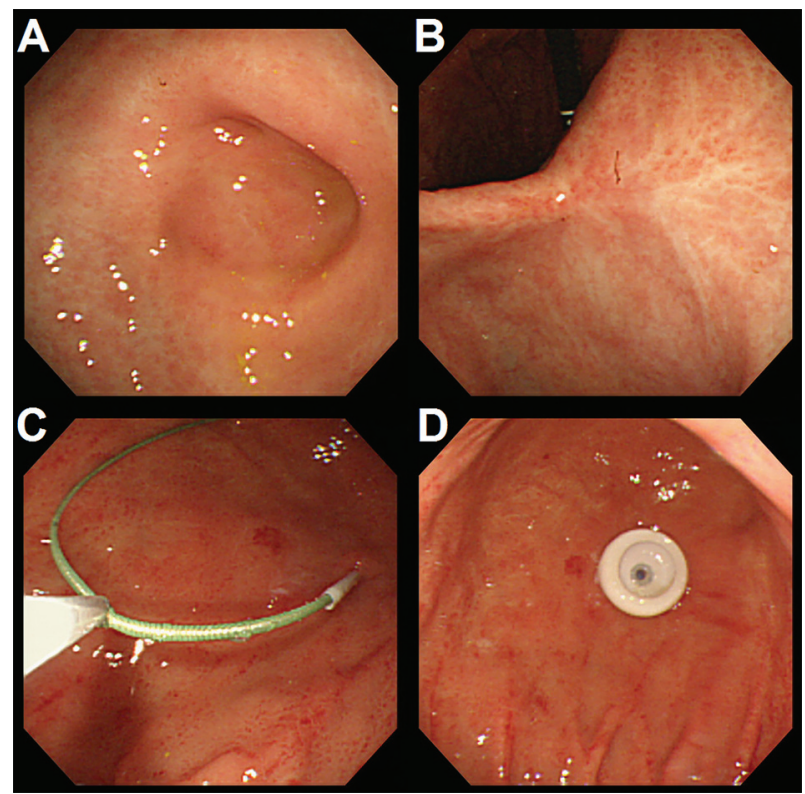

Figure 1. Endoscopic imaging before the procedure revealed a deformed duodenal bulb with moderate stenosis (A) and gastric ulcer scars (B). PEG was performed using the push technique on the anterior wall slightly towards the greater curvature of the stomach $(C, D)$. 


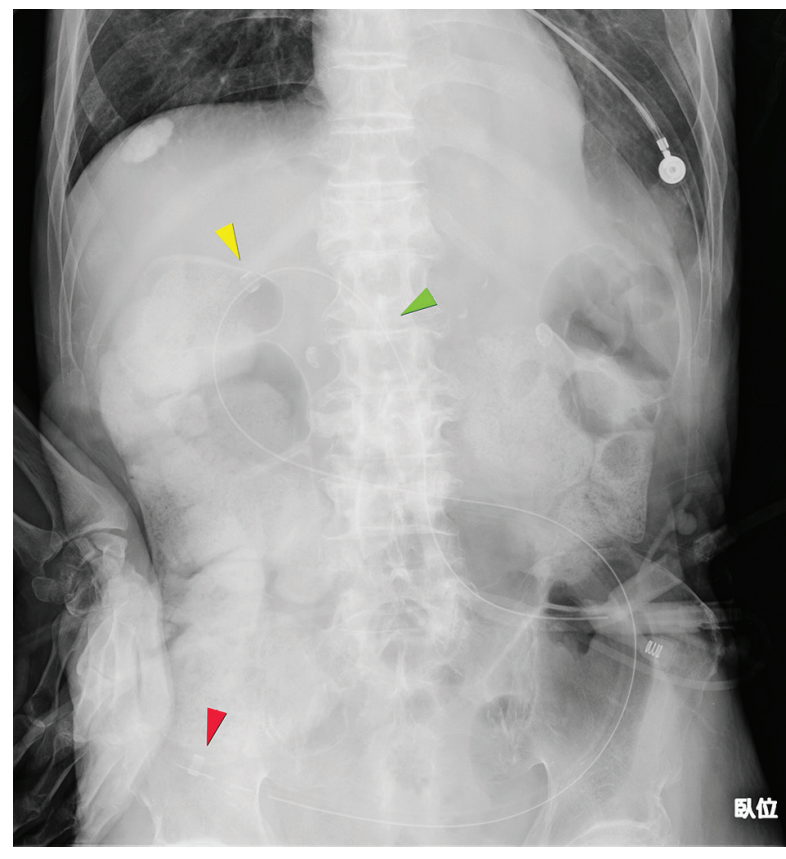

Figure 2. Abdominal X-ray taken after PEG-J tube placement.

PEG was performed using the push technique on the anterior wall slightly towards the greater curvature of the stomach (Fig. 1C, D).

Although the procedure ended uneventfully, emesis of liquid infusion into the gastrostomy tube occurred the following day, causing aspiration, asphyxiation and cardiac arrest. Cardiopulmonary resuscitation was successful and the patient received mechanical ventilation and total parenteral nutrition until he was stable. After receiving intensive care, the patient's condition stabilized and he was weaned from mechanical ven- tilation on day 70 after PEG procedure. After multidisciplinary consultation and discussion, the legal guardian chose enteral nutrition via PEG-J over total parenteral nutrition (TPN) via a central venous access port.

PEG-J was performed on day 83 (post-PEG) using a 20-Fr size all-silicone jejunal tube with gastric decompression function (Cliny PEG-J Catheter by Create Medic Co., Ltd, Yokohama, Japan). The procedure was performed in an interventional radiology suite with the use of fluoroscopy. An ultrathin endoscope was inserted into the gastric lumen through the gastrostomy puncture site after removal of the PEG tube and the PEG-J tube was placed so that the tip of the tube goes beyond the ligament of Treitz. Figure 2 shows an abdominal X-ray image taken after the procedure, revealing the PEG-J tube's position after placement. The green arrowhead indicates the entry point of the PEG-J tube through the gastrostomy puncture site, the yellow arrowhead points to the radiopaque marker before the pylorus and the red arrowhead shows the tip of the PEG-J tube in the jejunum.

Tube feeding resumed in a gradual manner until gravitycontrolled drip feeding was achieved. As shown in Figure 3, improvement of nutritional biomarkers such as total lymphocyte count (TLC), serum cholinesterase (ChE), total cholesterol (TC), triglyceride (TG), total iron binding capacity (TIBC), total protein (TP) and albumin levels (Alb) after PEG-J procedure was observed. After achieving stable tube feeding, the patient was discharged without further complications.

\section{Discussion}

Tube feeding can be initiated using nasogastric (or nasojejunal) tubes but for long-term enteral nutrition, percutaneous routes are always preferable. Although PEG is the percutaneous route of choice in most cases, feeding-related adverse events such as aspiration of gastric feed are an obstacle to successful enteral

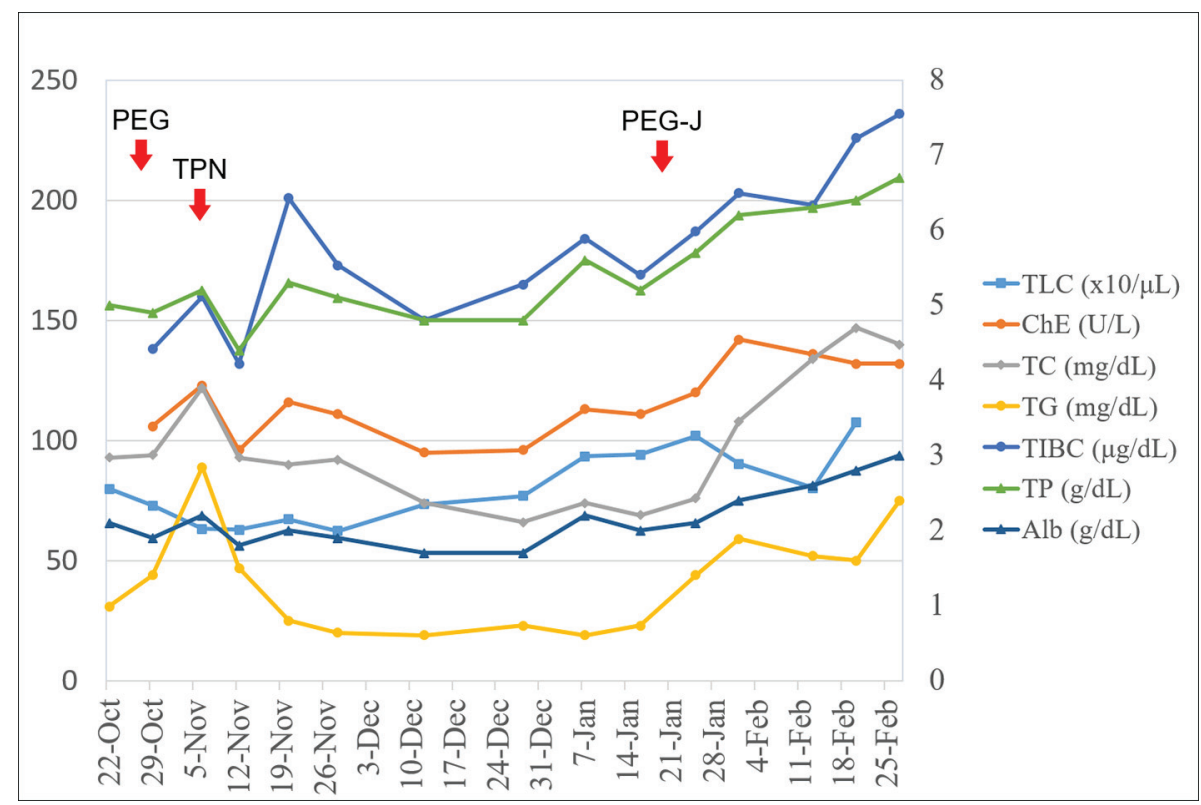

Figure 3. Improvement in nutritional biomarkers after PEG-J procedure. 
feeding. Semi-solid feeding instead of conventional liquid feed may help reduce the incidence of gastroesophageal reflux but may not be effective in cases with gastric outlet obstruction or stenosis [10].

By circumventing gastric passage during enteral nutrition and improving the drainage of gastric secretions via decompression holes, jejunal feeding through PEG-J may help prevent feeding-related adverse events encountered during PEG feeding $[3,4]$. Currently, instead of using jejunal extension tubes placed through PEG tubes, PEG-J can also be performed using large-bore gastrojejunal tubes (up to $24 \mathrm{Fr}$ size) that is placed directly through the PEG puncture site with or without the aid of endoscopy $[9,11]$.

As shown in this case, PEG-J can help improve the delivery of enteral nutrition when PEG feeding is unsuccessful due to feeding-related adverse events. With the aid of gastric decompression holes, excessive gastric secretions during jejunal feeding can also be removed simultaneously. On whether PEG-J should have been the method of choice from the beginning in this patient, this is open to discussion. Although retrospectively, the answer would be yes, it should be noted that the patient was tube-fed via a nasogastric route before PEG procedure, indicating that gastric feeding was reasonably tolerated. Transition to TPN via a central venous access port is also an option given to legal guardians of cases such as this. However, it not only exposes patients to adverse events such as catheterrelated blood stream infections but may also increase the cost of nutrition provision [12].

\section{Conclusion}

Tube feeding-related adverse events in PEG patients can be challenging and may delay or terminate enteral nutrition even in patients with intact gut function. Although PEG-J may not overcome all feeding-related adverse events, it can help maintain enteral feeding in patients who would otherwise be indicated for TPN. Since enteral nutrition is the route of choice when gut integrity is intact, PEG-J is an alternative worth exploring when encountering gastric feeding-related adverse events such as aspiration pneumonia. It should also be considered as the method of choice when attempting long-term enteral tube feeding in patients with significant gastric scarring or duodenal stenosis.

\section{Conflicts of Interest}

None.

\section{References}

1. DeLegge MH. Percutaneous endoscopic gastrostomy. Am J Gastroenterol. 2007;102(12):2620-2623.

2. Suzuki Y, Tamez S, Murakami A, Taira A, Mizuhara A, Horiuchi A, Mihara C, et al. Survival of geriatric patients after percutaneous endoscopic gastrostomy in Japan. World J Gastroenterol. 2010;16(40):5084-5091.

3. Zhang Z, Xu X, Ding J, Ni H. Comparison of postpyloric tube feeding and gastric tube feeding in intensive care unit patients: a meta-analysis. Nutr Clin Pract. 2013;28(3):371-380.

4. Adams GF, Guest DP, Ciraulo DL, Lewis PL, Hill RC, Barker DE. Maximizing tolerance of enteral nutrition in severely injured trauma patients: a comparison of enteral feedings by means of percutaneous endoscopic gastrostomy versus percutaneous endoscopic gastrojejunostomy. J Trauma. 2000;48(3):459-464; discussion 464-455.

5. Shike M, Schroy P, Ritchie MA, Lightdale CJ, Morse R. Percutaneous endoscopic jejunostomy in cancer patients with previous gastric resection. Gastrointest Endosc. 1987;33(5):372-374.

6. Ponsky JL, Aszodi A. Percutaneous endoscopic jejunostomy. Am J Gastroenterol. 1984;79(2):113-116.

7. Fan AC, Baron TH, Rumalla A, Harewood GC. Comparison of direct percutaneous endoscopic jejunostomy and PEG with jejunal extension. Gastrointest Endosc. 2002;56(6):890-894.

8. Zopf Y, Rabe C, Bruckmoser T, Maiss J, Hahn EG, Schwab D. Percutaneous endoscopic jejunostomy and jejunal extension tube through percutaneous endoscopic gastrostomy: a retrospective analysis of success, complications and outcome. Digestion. 2009;79(2):92-97.

9. Adler DG, Gostout CJ, Baron TH. Percutaneous transgastric placement of jejunal feeding tubes with an ultrathin endoscope. Gastrointest Endosc. 2002;55(1):106-110.

10. Nishiwaki S, Araki H, Shirakami Y, Kawaguchi J, Kawade N, Iwashita M, Tagami A, et al. Inhibition of gastroesophageal reflux by semi-solid nutrients in patients with percutaneous endoscopic gastrostomy. JPEN J Parenter Enteral Nutr. 2009;33(5):513-519.

11. Melvin W, Fernandez JD. Percutaneous endoscopic transgastric jejunostomy: a new approach. Am Surg. 2005;71(3):216-218.

12. Ott L, Annis K, Hatton J, McClain M, Young B. Postpyloric enteral feeding costs for patients with severe head injury: blind placement, endoscopy, and $\mathrm{PEG} / \mathrm{J}$ versus TPN. J Neurotrauma. 1999;16(3):233-242. 